\title{
Addressing the Hidden Terminal Problem for Heterogeneous Coexistence between TDM and CSMA Networks in White Space
}

\author{
Kaigui Bian, Member, IEEE, Jung-Min "Jerry" Park, Senior Member, IEEE, Lin Chen, \\ and Xiaoming Li, Senior Member, IEEE
}

\begin{abstract}
Cognitive radio (CR) technologies have led to several wireless standards (e.g., IEEE 802.11af and IEEE 802.22) that enable secondary networks to access the TV white-space (TVWS) spectrum. Different unlicensed wireless technologies with different PHY/MAC designs are expected to coexist in the same TVWS spectrum-we refer to such a situation as heterogeneous coexistence. The heterogeneity of the PHY/MAC designs of the coexisting CR networks can potentially exacerbate the hidden terminal problem. This problem cannot be addressed by the conventional handshaking/coordination mechanism between two homogeneous networks employing the same radio access technology (RAT). In this paper, we present a coexistence protocol, called Spectrum Sharing for Heterogeneous Coexistence (SHARE), that mitigates the hidden terminal problem for the coexistence between two types of networks: one that employs a TDM-based MAC protocol and one that employs a CSMA-based MAC protocol. Specifically, SHARE utilizes beacon transmissions and dynamic quiet periods to avoid packet collisions caused by the hidden terminals. Our analytical and simulation results show that SHARE reduces the number of packet collisions and guarantees weighted-fairness in partitioning the spectrum among the coexisting secondary networks.
\end{abstract}

\section{INTRODUCTION}

There is active participation by industry stakeholders in the ongoing standardization of TV white-space (TVWS, or fallow TV spectrum) technologies that utilize cognitive radio (CR) technology. In this paper, we use the term "CR network" to denote a CR-enabled wireless network operating over TVWS. Ongoing or completed standardization efforts include IEEE 802.22 [15], IEEE 802.11af (WiFi over TVWS or WhiteFi [1]), ECMA 392 (WPAN over TVWS) [6], etc. These standards will open the doors to scenarios where different unlicensed wireless technologies coexist in the same TVWS spectrum-we refer to such a situation as heterogeneous coexistence. TVWS is free of primary users' activities for secondary wireless networks' access, and information regarding the fallow TV spectrum can be retrieved from the TVWS databases [21]. In this paper, we focus on the coexistence between heterogeneous secondary wireless networks, and the

This work was partially supported by NSFC under grant number 61201245 , Beijing Natural Science Foundation (4143062), and US NSF grants of CNS0746925 and IIP-1265886.

Kaigui Bian, Lin Chen, and Xiaoming Li are with the Institute of Network Computing and Information Systems, School of EECS, Peking University, Beijing, China (email: \{bkg, abratchen, 1xm\}@ @ku.edu.cn). Jung-Min "Jerry" Park is with the Bradley Department of Electrical and Computer Engineering, Virginia Tech, Blacksburg, VA, USA (email: jungmin@vt.edu). interplay between primary users and secondary networks is out of scope of this paper.

Recognizing the importance of heterogeneous coexistence, the IEEE 802.19 Working Group has taken actions to address this issue, and recently formed a new task group (TG), i.e., the 802.19.1 TG. The 802.19.1 TG is chartered with the specific task of developing a standard for TVWS coexistence methods. The 802.19.1 standard is expected to address not only spectrum sharing but also channel sharing. With the expected proliferation of unlicensed TVWS devices, the number of available channels in TVWS is expected to be insufficient to provide a separate channel for each wireless technology or network in crowded spectrum environments, such as metropolitan areas. Such environments require a finegrained channel sharing mechanism to minimize co-channel interference among neighboring heterogeneous CR networks.

In wireless networks, when more than one transmitterreceiver pairs share a channel, the hidden terminal problem can occur. This problem occurs when a transmitter is visible from a receiver node, but hidden from (or out of the sensing range of) another transmitter that is visible from the same receiver. This leads to packet collisions at the receiver when the two transmitters send packets simultaneously. The hidden terminal problem in single-channel environments has been widely studied. In single-channel systems employing CSMA/CA, a handshaking procedure (i.e., using RTS/CTS control packets [18]) has been adopted to address the problem. For handling the hidden terminal problem in multi-channel wireless networks, some have proposed the use of a fixed control channel to facilitate a handshake procedure between two transceivers [29].

Unfortunately, the aforementioned handshaking procedures do not work when the hidden terminal problem is caused by heterogeneous coexistence. This is because the hidden terminal problem in heterogeneous coexistence is different from those mentioned above, and is due to the fact that the coexisting networks cannot understand each others' control messages because they use different air interfaces (i.e., PHY/MAC stacks). An example is shown in Figure 1 in which: an 802.22 network is coexisting with an 802.11af network. The 802.22's MAC protocol is TDM (time division multiplexing)-based with PHY resources allocated using OFDMA, while 802.11af relies on a contention-based CSMA protocol. Because the 802.22 base station (BS) and the 802.11af access point (AP) are hidden from each other, packets sent by the BS and 


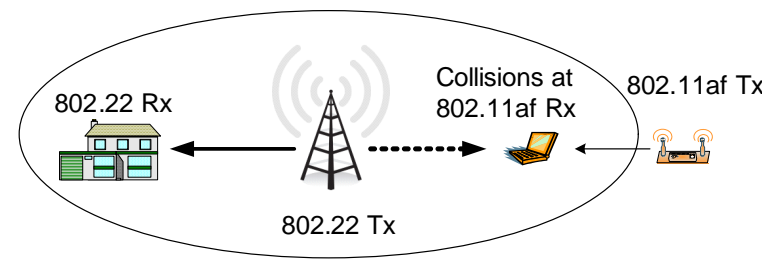

Fig. 1. An example of the hidden terminal problem caused by heterogenous coexistence.

the AP may collide at the 802.11af receiver node. Previous works have shown that enabling fair and efficient spectrum sharing is challenging in scenarios where a network with a contention-based MAC protocol (e.g., 802.11af) coexists with a network with a tightly-scheduled TDM-based MAC protocol (e.g., 802.22 or 802.16h) [7]-[9], [12], [28], [31]. ${ }^{1}$ We assume that the TDM device can distinguish a packet sent by a CSMA device from the background noise. This paper focuses on the particular type of heterogeneous coexistence between TDM and CSMA networks.

A mediated (a.k.a. centralized) coexistence solution can address the hidden terminal problem when coexisting networks can coordinate their operations via a "mediator". By interacting with each of the coexisting networks, the mediator can facilitate coexistence and prevent packet collisions caused by hidden terminals. The IEEE 802.19.1 TG has been focusing on such a coexistence scheme, where the 802.19.1 system [2], [13] serves as a mediator for coordinating spectrum sharing among heterogeneous CR networks. However, this approach requires every coexisting network to register with the mediator, and also requires the deployment of network infrastructure to support spectrum sharing. Hence, realizing a mediated coexistence scheme is expensive, and may not be practical for certain types of unlicensed TVWS networks-e.g., shortrange CSMA-based networks serving a few users.

An autonomous (a.k.a. uncoordinated) coexistence scheme is the only feasible approach when there are no means of coordination between the coexisting networks. Recently, a number of autonomous schemes have been proposed, in particular, to address the coexistence between WiFi and ZigBee. In those schemes, to achieve fair channel sharing between WiFi and ZigBee networks, busytone [35] and adaptive preamble [24] techniques have been proposed. These techniques increase the chance that a low-power ZigBee device is able to gain access to the channel in the presence of a higher-power WiFi network. The coexistence between IEEE 802.16 and WiFi has also been studied in the literature [3] as well as in the IEEE 802.16h standard [22]. For 802.16-WiFi coexistence, the 802.16 network employs quiet periods $(\mathrm{QP})$ to periodically vacate the current channel and thereby provide channel access opportunities for coexisting WiFi networks. Autonomous coexistence schemes focus on ensuring fair channel access for all of the coexisting networks, but fail to address the aforementioned hidden terminal problem. Henceforth, the CR

\footnotetext{
${ }^{1}$ IEEE 802.22 is the first worldwide wireless standard based on CR technology for utilizing TVWS in rural areas. The 802.22 standard prescribes incumbent protection techniques necessary for secondary users to operate in licensed TV bands, while 802.16 does not.
}

networks that employ a TDM-based MAC protocol will be referred to as TDM MAC networks, and those using a CSMAbased MAC protocol will be referred to as CSMA MAC networks.

In this paper, we present an autonomous coexistence scheme called Spectrum Sharing for Heterogeneous Coexistence SHARE. SHARE mitigates the packet collisions caused by the hidden terminal problem in a particular type of heterogeneous coexistence scenario-viz, coexistence of TDM and CSMA MAC networks (e.g., 802.22 and 802.11af networks). The hidden terminal problem in this heterogeneous coexistence scenario induces two types of packet collisions: (1) collisions at the receivers of a TDM MAC network that are caused by hidden transmitters of a CSMA MAC network; and (2) collisions at the receivers of a CSMA MAC network that are caused by hidden transmitters of a TDM MAC network. SHARE mitigates both types of packet collisions.

- To mitigate the first type of collisions, SHARE employs a beacon transmission mechanism that enables the receivers in a TDM MAC network to send beacon signals to prevent the hidden CSMA devices from accessing the shared channel while transmitters of the TDM MAC network occupy the channel.

- To mitigate the second type of collisions, SHARE prescribes a dynamic quiet period mechanism for the TDM MAC networks. This mechanism requires a TDM transmitter to dynamically determine the end point of its current quiet period (QP) in order to reduce the probability of packet collisions. The length of the quiet period is dynamically adjusted in order to maintain longterm weighted fairness in channel access between the coexisting TDM and CSMA networks.

Note that SHARE does not require coexisting networks to engage in direct coordination. Moreover, SHARE requires a minimal amount of change to the MAC protocols of the coexisting networks - specifically, only the algorithm for determining QPs in the TDM MAC protocol needs to be changed. Our analytical and simulation results show that SHARE reduces the number of packet collisions caused by the hidden terminal problem, and guarantees weighted fairness in spectrum sharing among the coexisting networks.

The rest of this paper is organized as follows: we provide background knowledge of existing heterogeneous coexistence schemes in Section II. In Sections III and IV, we give an overview of the system model and describe the problem and the key challenges to solve it. We present the two SHARE mechanisms in Section V. In Section VI, we evaluate the performance of SHARE using simulation results. We discuss related work in Section VII, and conclude the paper in Section VIII.

\section{TECHNICAL BACKGROUND}

As Figure 2 shows, heterogeneous coexistence schemes can be broadly classified into two categories: mediated coexistence and autonomous coexistence schemes. 


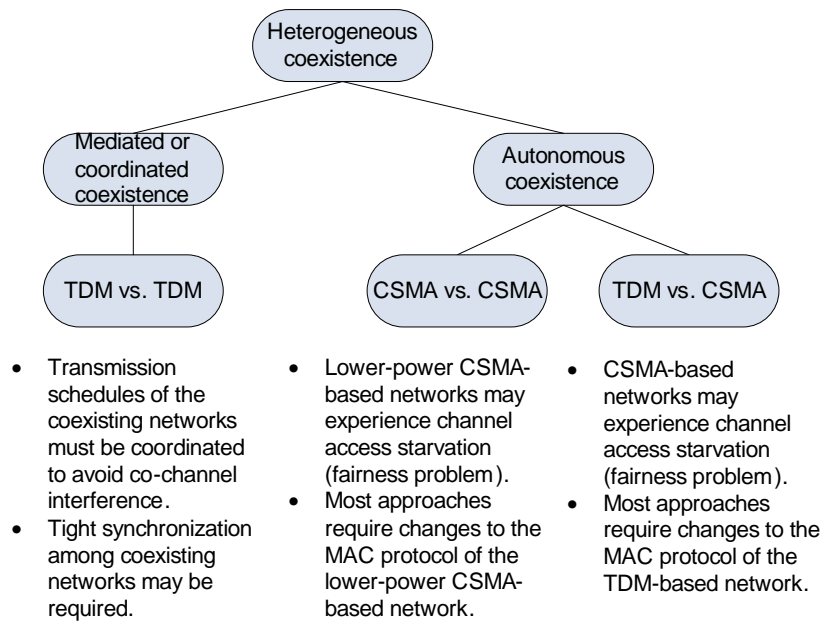

Fig. 2. Taxonomy of heterogeneous coexistence schemes.

\section{A. Mediated Coexistence Schemes}

When two or more TDM-based networks coexist in the same channel, each network's transmission schedule must be coordinated to avoid co-channel interference. Without such coordination, co-channel interference cannot be avoided since a TDM-based MAC protocol does not require the transmitter to sense the channel (for other nodes' signals) before transmission.

This type of coexistence scenario requires a mediated or coordinated coexistence scheme such as the ones enabled by the IEEE 802.19.1 system. An 802.19.1 system enables coexisting networks to coordinate their spectrum/channel sharing. It interacts with three external entities via backhaul connections [14]: a TVWS database, the TVWS device/network, and the Operator Management Entity (OME). Through the 802.19.1 system, coexisting TVWS networks can readily exchange control information through the interfaces of the 802.19.1 system to carry out collaborative channel sharing.

\section{B. Autonomous Coexistence Schemes}

For some coexistence scenarios, employing a mediated coexistence scheme is too expensive and may not be practical. Most of the existing work on heterogeneous coexistence focuses on solutions that can be categorized under the autonomous coexistence class. This class of solutions does not require coordination between the coexisting networks.

In the context of coexistence between heterogeneous CSMA networks, the fairness problem for low-power CSMA devices has garnered attention recently. An example is the coexistence between WiFi and ZigBee networks [11], [24], [35], [36].

In third type of coexistence scenario, CSMA MAC networks are at a disadvantage compared to TDM MAC networks, and may experience channel access starvation due to their listenbefore-talk approach for accessing spectrum. The 802.16h standard employs quiet periods $(\mathrm{QPs})$ to increase spectrum access opportunities for coexisting 802.11 networks. Specifically, an 802.16 network suspends its transmissions during a periodically-scheduled QP so that a coexisting 802.11 network can access the channel during those periods [3], [22]. The length of a QP is determined by the 802.16 network.

Note that the QP approach described above does not address the problem of packet collisions caused by hidden terminals. To the best of our knowledge, this paper presents the first study of this particular problem in the context of heterogeneous coexistence of TDM and CSMA networks.

\section{SYSTEM MODEL}

\section{A. Basic Assumptions}

We assume an opportunistic spectrum sharing environment, where TDM and CSMA MAC CR networks are co-located and operating over TVWS. Every CR network is composed of multiple TV band devices (TVBDs) and a CR-enabled network controller-e.g., a base station (BS) in 802.22 or an access point (AP) in 802.11af. In this paper, we assume that heterogeneous CR networks use the TVWS channel as the underlying channel model.

For detecting primary user signals, we assume that a CR network employs a hybrid method -i.e., using the information provided by a TVWS database as well as information gathered from spectrum sensing [2], [21]. Energy detection techniques are utilized for spectrum sensing. A CR network is able to obtain the list of channels that are free of primary user signals at its location by making inquiries to the TV database via the 802.19.1 interfaces.

In most deployment scenarios, the TDM networks operating in TVWS will need to be registered with a coexistence enabling system (a.k.a. coexistence mediator), such as an 802.19.1-compliant system, to minimize interference to other networks. We also assume that all TDM MAC networks synchronize their clocks via the 802.19.1 interfaces.

However, CSMA MAC networks are typically low-power networks, which service a few number of people and are managed by an individual or a small group. Because of the ad hoc nature of CSMA network deployments and their short range, it is plausible to assume that they are not registered with a coexistence mediator and do not recognize its commands. In this paper, we assume that all of the TDM networks are registered with an 802.19.1-compliant system, whereas CSMA networks are not registered.

\section{B. Quiet Period}

In TDM MAC networks, channel access occurs in scheduled blocks of timeslots; in CSMA MAC networks, channel access is contention based and there is no pre-determined schedule for channel access. In this paper, we define a "universal" superframe structure that is representative in TDM MAC networks with coexistence mechanisms (e.g., 802.22 or 802.16 networks). Time is divided into superframes, each superframe is divided into frames, and each frame contains a number of $f$ timeslots.

In 802.16h, a quiet period $(\mathrm{QP})$ that contains an integer number of frames is periodically scheduled [15], [22]. During a QP, the BS suspends its data transmissions to provide channel access opportunities for CSMA networks. Similarly, 
TABLE I

Notations.

\begin{tabular}{|c|c|}
\hline Notation & Definition \\
\hline$q$ & $\begin{array}{l}\text { The number of frames in a quite period (basic } \\
\text { QP length) }\end{array}$ \\
\hline$d$ & $\begin{array}{l}\text { The number of non-QP (data) frames in a } \\
\text { superframe }\end{array}$ \\
\hline$f$ & The number of timeslots in a frame \\
\hline$e_{i}$ & $\begin{array}{l}\text { The duration of the elapsed QP in the } i \text {-th } \\
\text { superframe }\end{array}$ \\
\hline$r_{i}$ & $\begin{array}{l}\text { The duration of the residual QP in the } i \text {-th } \\
\text { superframe }\end{array}$ \\
\hline$l_{d}$ & The mean length of the CSMA data packet \\
\hline$l_{a}$ & The length of the CSMA ACK packet \\
\hline$p$ & $\begin{array}{l}\text { The probability that the next CSMA packet } \\
\text { transmission can be finished in the residual QP }\end{array}$ \\
\hline$q_{i}$ & The number of frames in the $i$-th superframe \\
\hline $\begin{array}{l}f \text { time } \\
\text { slots }\end{array}$ & $\begin{array}{c}\text { TDM superframe contains } \\
(d+q) \text { frames }\end{array}$ \\
\hline TDM frame & \begin{tabular}{|l|l|l} 
TDM frame & TDM frame \\
\end{tabular} \\
\hline
\end{tabular}

Fig. 3. The superframe structure of TDM networks.

we define a QP at the end of every superframe, and we call non-QP frames as data frames.

Let $q$ denote the number of frames contained in a $\mathrm{QP}$, and let $d$ denote the number of data frames contained in a super frame. Thus, the number of frames in a superframe is $d+q$, and the number of time slots in a super frame is $(d+q) f$. A TDM network starts transmitting data immediately after the end of a QP (i.e., end of a superframe). The value of $q$ quantifies the number of frames that TDM networks can share with CSMA networks during a super frame. The values of $d$ and $q$ are predetermined collectively by the coexisting TDM MAC networks or determined by the coexistence mediator. The notations and their definitions are summarized in Table I. Figure 3 illustrates the superframe structure of a TDM MAC network. As the figure shows, during a TDM QP, the CSMA networks sense an idle channel and start to transmit CSMA packets.

\section{The Hidden Terminal Problem}

The hidden terminal problem can occur for all three types of heterogeneous coexistence scenarios, namely the coexistence between (1) two TDM systems, (2) two CSMA systems, and (3) a TDM and a CSMA systems.

The problems in the first two types of coexistence scenarios can be well addressed using existing solution introduced in Section II, and thus we focus on the third type of coexistence scenarios in this section. We identify two types of packet collisions caused by the hidden terminals when TDM networks coexist with CSMA networks.

\section{A. Collisions at the TDM Network Receiver}

This type of collisions occur when a TDM receiver is located within the transmission ranges of both the TDM and CSMA transmitters, but the two transmitters are hidden from

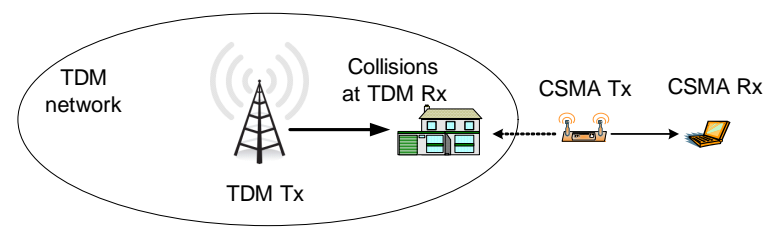

Fig. 4. Packet collisions at the TDM receiver due to the simultaneous transmissions by the TDM and the CSMA transmitters.

each other. Figure 4 shows an example where the CSMA transmitter's transmission causes packet collisions at the TDM receiver. To reduce this type of collisions, the TDM network has to prevent the CSMA transmitter from transmitting while the TDM transmitter is transmitting.

A straightforward solution to mitigate packet collisions in this scenario is to require the TDM receiver to emit beacon signals during a small time fraction at the beginning of every time slot. We call this time fraction as the beaconing fraction of a time slot. Here, we assume that the TDM receiver is within the CSMA transmitter's sensing range-i.e., the CSMA transmitter can sense the TDM receiver's beacon signals. The beacon signal's presence in the channel will cause the CSMA transmitter to suspend transmissions. It is reasonable to assume that the coexistence enabling system (e.g., 802.19.1 system) mandates the use of beacon signals by the TDM receivers to facilitate coexistence, since the TDM MAC networks are registered.

During the beaconing fraction of a time slot, the TDM transmitter stops transmitting, and the TDM receiver emits beacon signals such that the coexisting CSMA transmitter can detect the beacons and refrain from transmitting in the channel. As a result, the collision-free receptions at the TDM receivers can be guaranteed. Requiring the TDM receiver to transmit beacons can be a costly overhead, and thus should be required only when its benefits outweigh the costs. In Section V, we present a beacon transmission mechanism, which enables the TDM receiver to dynamically determine whether to transmit or not transmit the beacons depending on the channel conditions.

\section{B. Collisions at the CSMA Network Receiver}

This type of collisions occur when the CSMA receiver is located within the transmission ranges of both the TDM and CSMA transmitters but the two transmitters are hidden from each other. In this scenario, packet collisions occur because the TDM transmitter initiates transmission before the CSMA transmitter has finished transmitting its data packets.

A specific example of this scenario is shown in example in Figure 5. In the figure, the first TDM frame transmitted by the TDM transmitter in the $(N+1)$-th superframe collides with the CSMA transmitter's ongoing packet transmissions which started in the QP of the $N$-th superframe and has continued on past the QP. In this situation, requiring the CSMA receivers to use beacons is not a plausible solution, because, they are not under the control of the 802.19.1 system.

During a QP, a TDM transmitter suspends its transmission, and it terminates the QP at the scheduled end time point by 


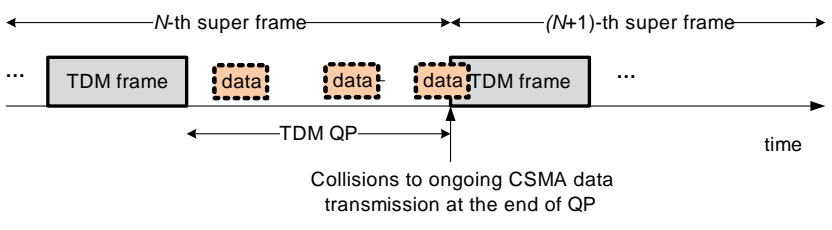

Fig. 5. Collisions at the end of the $N$-th frame's QP. Dotted-line rectangles represent the CSMA data packets.

transmitting a prescribed number of beacons. After transmitting a prescribed number of beacons, the TDM transmitter terminates the current QP and starts the TDM frame of the next superframe. Note that the TDM transmitter simply terminate the QP at the scheduled time point, and it will not wait until the ongoing transmission for CSMA data and/or ACK packets to be finished.

In Section $\mathrm{V}$, we propose a collision avoidance algorithm for the TDM networks that mitigates this type of collisions. This algorithm allows the TDM transmitter to dynamically decide whether to make an early termination of QP by immediately transmitting beacons when detecting a CSMA ACK packet before the original end point of the QP. As described in [11], the TDM transmitter simply assumes that undecodable short packets are the ACK packets sent by the CSMA receiver.

The beacons are transmitted to compel the CSMA receiver to stop accessing the channel after sensing these beacons (or stop responding to the CSMA transmitter). An example is shown in Figure 6. In the figure, an early termination of the original QP occurs, and the first TDM frame of the $(N+1)$ th superframe (advanced frame) started immediately after the transmission of the beacons.

It should be noted that the occurrence of an advanced frame will not change the original schedule of TDM transmissions. Instead, the TDM transmitter only needs to schedule transmissions during the newly-obtained advanced frame that substitutes the residual QP (the residual QP is replaced by the advanced TDM frame.)

\section{The Weighted Fairness Problem}

To help quantify the notion of fairness in co-channel sharing among multiple networks, we define a metric called access time ratio (ATR). The ATR for a network is the ratio between the channel access time of the network to the channel access time of all the other networks combined.

When a TDM network coexists with one or more CSMA networks, the ATR for the TDM network can be expressed as $\frac{d}{d+q}$, using the notations given in Table I. We assume that the values of $d$ and $q$ are predetermined by the coexistence mediator to ensure some notion of fairness in the coexisting networks' channel access. When the TDM network achieves an ATR value of $\frac{d}{d+q}$, we say that the channel sharing is weighted fair with respect to that network. Note that because the CSMA networks are not registered with the coexistence mediator, it is implausible to assume that the coexistence mediator would try to ensure fairness for the CSMA networks in a definitive sense; instead, it would likely try to ensure adequate channel access time for the CSMA networks based on predetermined

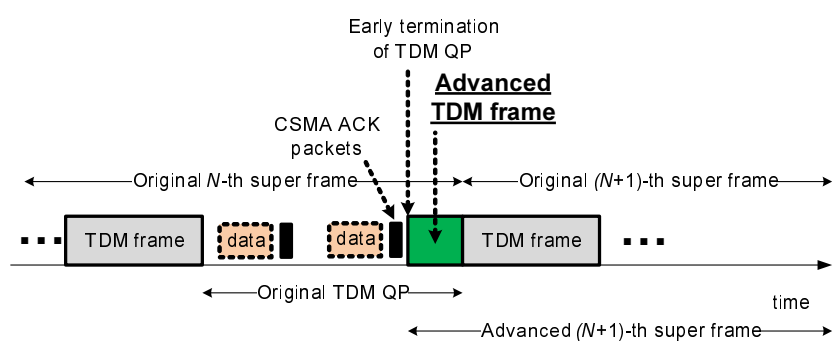

Fig. 6. The QP in the $N$-th superframe is adjusted dynamically to mitigate packet collisions. Black rectangles represent the CSMA ACK packets.

spectrum policies or rules, and thus ensure a policy-defined notion of fairness.

When the values of $d$ and $q$ are fixed, ensuring weightedfair channel sharing is trivial, since the registered TDM networks simply need to use those values set by the coexistence mediator. However, as discussed previously, one method of mitigating collisions at the CSMA receiver is to dynamically adjust the length of QPs. This approach affects the ATR of the coexisting networks, and in turn affects fairness. In Section V, we discuss the tradeoff between collision avoidance and weighted fairness in the context of the SHARE protocol.

\section{THE SHARE PROTOCOL}

SHARE prescribes a beacon transmission mechanism and a dynamic quiet period mechanism to mitigate the above two types of collisions that are introduced by the hidden terminal problem.

\section{A. Beacon Transmission by TDM Receiver}

We consider an 802.22 WRAN co-located with an 802.11af WLAN, both sharing the same TVWS channel. To mitigate the first type of collisions, SHARE supports two modes at the TDM receiver: beaconing and non-beaconing modes. It can switch from one mode to the other depending on the channel conditions, and this procedure of switching contains two steps:

1) SHARE performs channel evaluation to determine when to switch from one mode to the other. The TDM receiver measures the received SIR, estimates the channel capacity in the two modes, and makes a decision of which mode to operate in.

2) Then, the TDM receiver will notify the TDM transmitter if it decides to switch to the beaconing mode. In the beaconing mode, the TDM transmitter has to stop transmitting in the beaconing duration.

Beaconing by TDM receivers will incur additional overhead and waste the channel time. That is why we need a dynamic switch between the two modes to balance the tradeoff between the performance loss due to interference caused by CSMA packets, and the beaconing overhead.

First, SHARE needs to calculate the channel capacity in each of the two modes. By estimating the channel access time and packet error rate, we express the capacity of the shared channel, $C$, as follows.

$$
C=\left(1-\frac{u}{t}\right) \cdot(1-\epsilon)
$$


where $t$ is the length of a time slot, $u$ is the duration of beaconing in a time slot, and $\epsilon$ denotes the packet error rate on the shared channel at the TDM receiver. In (1), $\left(1-\frac{u}{t}\right)$ represents the ratio of channel access time used for nonbeaconing transmission and the length of a time slot; and $(1-\epsilon)$ shows the rate of successful packet reception given possible packet errors. The packet error can be caused by a few factors, such as noise, interference, fading, etc.

The reduction of channel capacity can be caused by two factors in the considered scenarios of heterogeneous coexistence, namely the beaconing duration, and the inter-network interference. In the beaconing mode, the beaconing duration, $u$, is the major cost that may lower the channel capacity, and the inter-network interference can lead to a high packet error rate (i.e., a low channel capacity according to (1)) in the nonbeaconing mode. The effect of fading may vary with time, geo-locations, or frequency, and it is independent of whether the beaconing mode is used.

Let $\gamma$ denote the maximum achievable SIR perceived by the TDM receiver on the shared channel. In [27], Shellhammer describes a way of estimating $\epsilon$ based on $\gamma$ : the symbol error rate (SER), $\rho$, can be estimated based on $\gamma$; then, $\epsilon$ can be calculated based on the SER. If the modulation is BPSK, the SER $\rho=Q[\sqrt{2 \gamma}]$, where the function $Q(\cdot)$ is the integral of the tail of a normalized Gaussian probability density function [23]. The packet error rate in a packet of $m$ symbols is the probability that at least one symbol is incorrect,

$$
\epsilon=1-(1-\rho)^{m} \text {. }
$$

1) Channel capacity in beaconing mode: To avoid the first type of collisions, the TDM receiver is allowed to block the medium by emitting beacon signals in every time slot. The capacity of the shared channel in this mode can be expressed as

$$
C_{b}=\left(1-\frac{u}{t}\right) \cdot(1-\epsilon) \text {. }
$$

The CSMA transmitter could sense an idle channel during a time period of $(t-u)$ in every TDM time slot. To successfully block the medium, the TDM receiver needs to guarantee that the value of $(t-u)$ is less than the time for channel clear assessment (CCA) in CSMA networks. ${ }^{2}$

Assume the noise source is additive white Gaussian noise (AWGN) $N_{0}$, and the signal-to-noise ratio is represented as $E_{s} / N_{0}$, where $E_{s}$ denotes the signal energy in a symbol. That is,

$$
\gamma=\frac{E_{s}}{N_{0}} .
$$

Given the modulation, the TDM MAC network is able to estimate $C_{b}$ using $\gamma$.

2) Channel capacity in non-beaconing mode: If the feature of beacons is disabled, the TDM receiver may experience the first type of collision when there is a nearby CSMA transmitter that is hidden from the TDM transmitter. The channel capacity in the non-beaconing mode can be expressed as

$$
C_{n}=\left(1-\frac{u}{t}\right) \cdot(1-\epsilon)=1-\epsilon .
$$

\footnotetext{
${ }^{2}$ The time for CCA in WiFi networks is $28 \mu s$ [33].
}

The fundamental principal of this approach in the nonbeaconing mode is to equate the interference power at the TDM receiver, after the receive filter, to the equivalent noise power after the receive filter.

Given the AWGN $N_{0}$, the signal energy in a symbol of period $T$ is related with the symbol power $P_{s}=\frac{E_{s}}{T}$. Let $B$ denote the noise equivalent bandwidth of the receive filter for a symbol period of $T$, and $B=\frac{1}{T}$. The noise power after the receiver filter is given by $P_{n}=\frac{N_{0}}{B}$. The ratio $E_{S} / N_{0}$ can be expressed in terms of the signal power and the noise power after the receiver,

$$
\frac{E_{s}}{N_{0}}=\frac{P_{s} T}{P_{n} T}=\frac{P_{s}}{P_{n}},
$$

Since the TDM receiver has the same TVWS channel model as the interferer (CSMA transmitter), the noise power after the receive filter is equal to the value of the interference signal, $P_{I}^{r}$, after the receive filter [27], i.e., $P_{n}=P_{I}^{r}$. Therefore,

$$
\gamma=\frac{E_{s}}{N_{0}}=\frac{P_{s}}{P_{I}^{r}},
$$

and $C_{n}$ can be accordingly estimated.

3) An approach of switching between two modes: In SHARE, the non-beaconing mode over the shared mode is attempted first. If the maximum achievable SIR on the channel is not sufficiently high, SHARE prescribes the beaconing mode for TDM networks to block the medium and prevent CSMA devices' access to the channel. We propose one possible approach for determining when to enable the beaconing mode.

SHARE determines which mode for the the TDM receiver to operate in, for a given channel, by comparing $\gamma$ with the required SIR threshold parameter $\gamma^{*}$. To obtain the value of $\gamma^{*}$, we need to first solve for $\epsilon$ in the equation $C_{b}=C_{n}$; and then find the SIR value corresponding to the solved $\epsilon$, which is used as the value of $\gamma^{*}$.

1) If $\gamma \geq \gamma^{*}$, the TDM receiver operates in the nonbeaconing mode. The benefits of non-beaconing mode (e.g., low control overhead) outweigh the benefits of beaconing (e.g., no collisions).

2) If $\gamma<\gamma^{*}$, the TDM receiver operates in the beaconing mode. The benefits of beaconing mode outweigh the benefits of non-beaconing mode.

\section{B. Dynamic Quiet Period at TDM Transmitter}

In SHARE, the TDM transmitter chooses the appropriate starting time point for TDM transmission in order to avoid overlapped TDM and CSMA transmissions, and it starts occupying the channel immediately after the transmission of a whole CSMA packet, and before the transmission of the next CSMA packet.

SHARE is built on top of the super frame structure of TDM MAC networks, and it adopts an innovative way of dynamically determining the length of the quiet period, which reduces the second type of collisions as well as maintains the weighted fairness for the TDM MAC network in channel access. SHARE employs the following algorithms to achieve these objectives. 
- Collision avoidance algorithm. The TDM transmitter monitors the data traffic during the QP, and captures the ACK packets emitted by CSMA networks. To avoid collisions, the TDM transmitter is allowed to terminate the QP immediately after detecting an ACK packet. This will lead to a shortened QP in the current super frame, and an advanced frame in the next super frame, which hurts the short-term weighted fairness for the TDM MAC network.

- Weighted fairness maintenance algorithm. By counting the number of time slots lost in previous shortened QPs, the TDM transmitter is able to determine whether it needs to increase the length of the next QP such that the longterm fairness can be maintained.

Next, we introduce the two algorithms in details.

1) Collision Avoidance: In either saturated or unsaturated WLANs, the aggregated traffic pattern (the inter-arrival time between WLAN packets) approximates a Poisson distribution [5], [35]. The experimental results in [11] show that the inter-arrival time of WLAN frame-clusters fits a Pareto model. In this paper, we assume that the packet arrival of the WLAN follows a Poisson distribution. Let $\lambda$ denote the WLAN data packets' arrival rate, and thus the mean inter-arrival time is $1 / \lambda$.

Definition 1: When the TDM network enters a scheduled QP, we define

- the elapsed $Q P$ as the time duration from the start of the QP to the current time point;

- and the residual $Q P$ as the time duration from the current time point to the expected end of the QP.

Figure 7 illustrates the elapsed QP and residual QP.

To avoid collisions to the potential hidden CSMA receiver at the end of the QP (defined as the second type of collisions), the TDM MAC network (or TDM transmitter) has to make a decision upon detecting a CSMA ACK packet: Is the residual QP long enough for completing another CSMA packet transmission?

1) If the answer is "yes", the TDM network will keep silent and wait for the next CSMA packet arrival (i.e., the next detected ACK).

2) If the answer is "no", the TDM network should terminate the QP by immediately starting TDM transmissions (or beacon transmissions) such that the CSMA receiver would refrain from operating in the channel.

As a result, SHARE enables the TDM MAC network to autonomously terminate the QP at an "appropriate" time point.

Let $e_{i}$ denote the duration of the elapsed QP and $r_{i}$ denote the duration of the residual QP, in the $i$-th super frame, both of which are on a time slot basis. Let $l_{d}$ and $l_{a}$ denote the mean length of WLAN's data packets and the length of ACK packet respectively. Recall that the number of time slots in a frame is $f$. Let $p$ denote the probability that the next CSMA packet transmission can be finished in the residual QP, $r_{i}$. Upon detection of an ACK packet, the length of the residual QP is $r_{i}$, and the TDM network calculates the probability $p$.

- When $r_{i}<l_{d}$, the residual QP is smaller than the mean length of a WLAN data packet, and thus $p=0$.

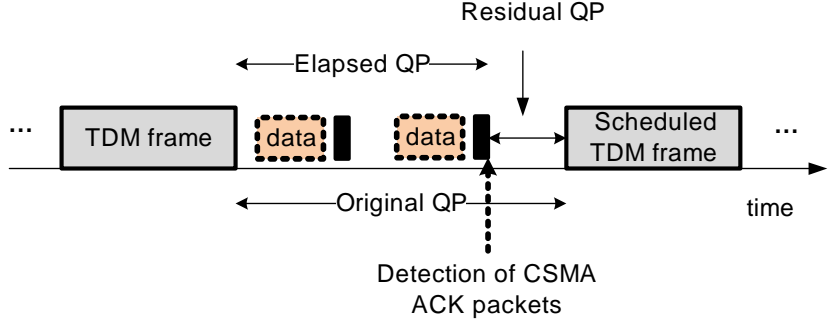

Fig. 7. Illustration of the elapsed QP and residual QP in a superframe of the TDM MAC network.

- When $r_{i} \geq l_{d}$, the residual QP is longer than the mean length of a WLAN data packet, and the next CSMA packet transmission can be finished in the residual QP, only if the packet arrives in next $\left(r_{i}-l_{d}\right)$ slots.

$$
\begin{aligned}
p & =\mathbb{P}\left\{\text { CSMA pkt arrival in next }\left(r_{i}-l_{d}\right) \text { slots }\right\} \\
& =1-\mathbb{P}\left\{\text { no CSMA pkt arrival in next }\left(r_{i}-l_{d}\right) \text { slots }\right\} \\
& =1-e^{-\lambda\left(r_{i}-l_{d}\right)} .
\end{aligned}
$$

Decision rule of dynamic QP mechanism. Based on the calculated value of $p$, the TDM network is able to decide whether to terminate the QP upon detection of CSMA ACK packets, using the following decision rule. The pseudo-code in Algorithm 1 illustrates this process, in which $q_{i}$ denotes the length of $Q P$ (on a frame basis) in the $i$-th superframe, and the initial value $q_{1}=q$. How to dynamically update the value of $q_{i}$ will be described in Algorithm 2 later.

1) When $e_{i}<q_{i} f$, the QP of the current superframe has not finished. Upon the detection of a CSMA ACK packet,

a) the TDM network predicts that the residual QP is insufficiently long for completing a next CSMA packet transmission if $p<\tau$. Then, it terminates the QP by immediately transmitting beacons;

b) the TDM network predicts that the residual QP is long enough for completing a next CSMA packet transmission if $p \geq \tau$. Thus, the TDM transmitter waits for the next ACK packet without sending any beacons.

$\tau$ denotes the threshold that represents the expected probability that the next CSMA packet transmission can be finished in the residual $Q P$.

2) When $e_{i}=q_{i} f$ (i.e., the residual QP $r_{i}=0$ ), it means that the elapsed QP length exceeds the original length of QP in the $i$-th superframe, and the TDM network has to terminate the QP immediately even without detecting any ACK packet. In unsaturated CSMA networks, the waiting time before a CSMA packet arrival might be very long, which degrades the channel utilization. This step prevents the case that the waiting time is longer than the length of the current QP.

The feasibility of collisions. By executing the collision avoidance algorithm under the hidden terminal situation (as shown in Figure 1), the TDM network may terminate the QP earlier than the time point of the original end. When the early termination of a QP happens, an advanced TDM frame starts, and the CSMA receiver will be prevented from responding 


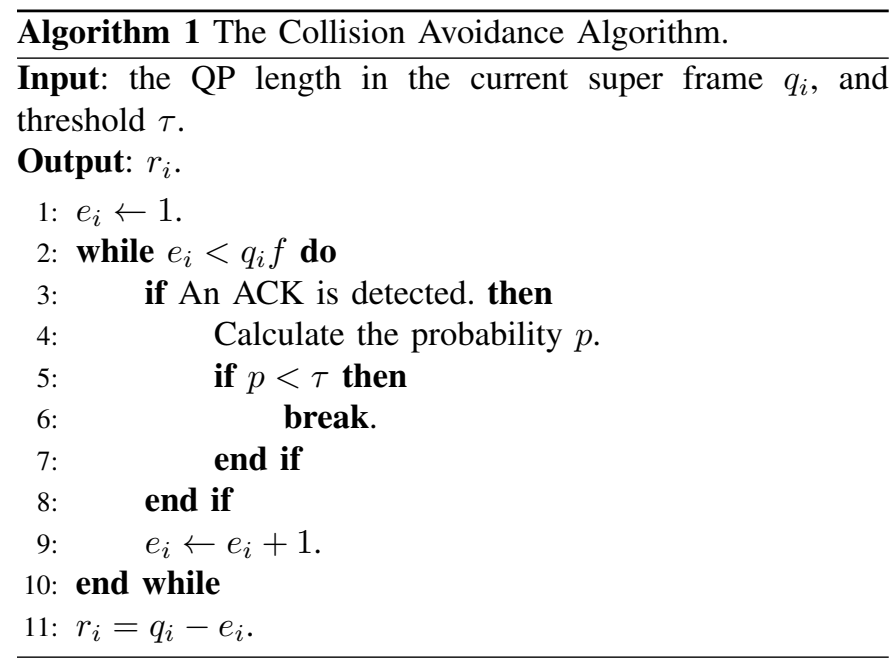

ACK to the CSMA transmitter over the channel. After a number of re-transmission attempts, the CSMA transmitter will defer its transmission until the next QP when the channel is idle. In other words, the CSMA receiver will not accept any data packets from the CSMA transmitter after the end of the early-terminated QP, and thus there is no collision to CSMA packet receptions since then.

2) Weighted-fairness Maintenance: An advanced TDM frame caused by the collision avoidance algorithm will lead to shortened QPs, and hurt the weighted-fairness for TDM MAC networks. To address this problem, the TDM MAC network has to correct the access time ratio to be $\frac{d}{d+q}$ by adding more time slots in the future QP.

The pseudo-code in Algorithm 2 illustrates an approach to update the dynamic QP length $q_{i}$ with purpose of weightedfairness maintenance, where $i>1$ and $q_{1}=q$. We call $q$ as the basic QP length. At the beginning of the $i$-th superframe, the TDM MAC network calculates the QP length $q_{i}$ in the current super frame as follows.

1) The TDM MAC network counts the number of time slots that are lost in the previous QP: the residual QP in the $(i-1)$-th superframe, $r_{i-1}$, represents such a number, which can be obtained from the output of Algorithm 1 executed in the $(i-1)$-th superframe. To maintain the weighted-fairness, the TDM network has to add $r_{i-1}$ slots in one of its future QPs.

2) In the $i$-th super frame, the TDM MAC network is allowed to make a decision whether to extend the basic QP length by $k_{i}$ frames such that the time slots lost in previous QPs can be somewhat compensated. That is, the number of frames in the $i$-th QP is given by $q_{i}=q+k_{i}$.

3) To help making the decision, the TDM network maintains a counter, $\mathcal{R}_{i}$, that counts the number of time slots that need to be compensated in the $i$-th QP,

$$
\mathcal{R}_{i}=\sum_{j=1}^{i-1} r_{j}-\sum_{j=1}^{i-1} k_{j} f
$$

a) If $\mathcal{R}_{i} \geq f$, calculate

$$
k_{i}=\frac{\mathcal{R}_{i}-\left(\mathcal{R}_{i} \bmod f\right)}{f} .
$$

Then, the TDM MAC network extends its current QP by $k_{i}$ frames, i.e., $q_{i}=q+k_{i}$.

b) Otherwise, $k_{i}=0$ and $q_{i}=q$.

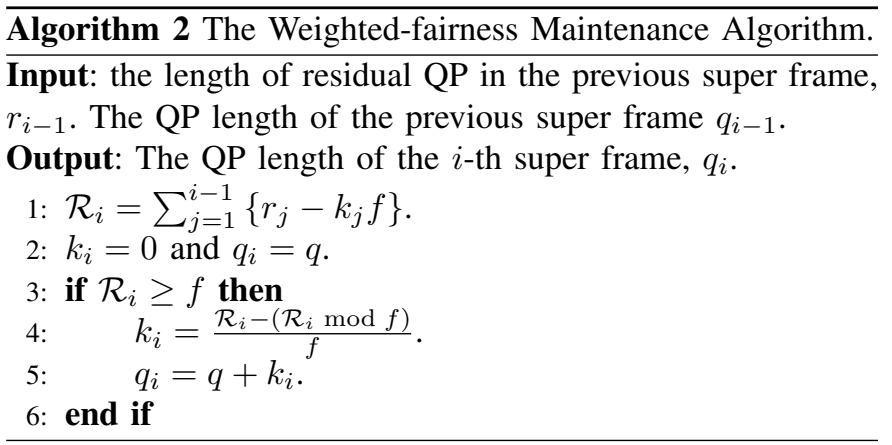

\section{Analysis}

Using analytical results, we show the superiority of the dynamic QP approach of SHARE over the fixed QP approach [15], [22]. We model the transmissions by the CSMA transmitter as a renewal process. Each renewal interval is the $\mathrm{QP}$, and the reward is the amount of time used for transmitting the CSMA packets without collision.

1) Collision probability:

Lemma 1: When the coexisting TDM and CSMA transmitters are hidden from each other, the probability of the second type of collisions incurred by the dynamic QP approach of SHARE is lower than that of the fixed QP approach.

Proof:

The fixed QP approach. In the $i$-th TDM super frame, all the TDM data frames have a duration of $d f$, and the $i$-th TDM QP is between the $i$-th and the $(i+1)$-th super frames. The TDM transmission may cause collisions to CSMA packets at the end of the QP. A CSMA packet that arrives in the last $l_{d}$ slots of the $i$-th QP will be collided by the TDM packet in the $(i+1)$-th super frame. In this approach, the collision probability, $p_{c}$, is equivalent to the probability that the CSMA packet arrival occurs before TDM transmissions finish-i.e., the CSMA packet arrival occurs in the last $l_{d}$ slots of the QP.

$$
p_{c}=\mathbb{P}\left\{\text { CSMA pkt arrival in last } l_{d} \text { slots }\right\}
$$

The dynamic QP approach. In this approach, upon detecting a CSMA ACK packet, the TDM MAC network is allowed to dynamically changes the end point of the QP. When the residual QP $r_{i}>l_{d}$,

- Case 1. Suppose the TDM network makes a decision of the early-termination of the QP upon an ACK detection. There is no collision in this case, and the collision probability in this case $p_{1}=0$.

- Case 2. Suppose the TDM network does not make a decision of the early-termination of the QP, and wait until the end of the scheduled QP. The collision probability in this case, $p_{1}$, is equivalent to the probability that the CSMA packet arrival occurs in the last $l_{d}$ slots of the QP. That is, $p_{2}=p_{c}$. 
The collision probability incurred by the dynamic QP approach,

$$
p_{c}^{\prime}=\mathbb{P}\{\text { Case } 1\} \cdot p_{1}+\mathbb{P}\{\text { Case } 2\} \cdot p_{2}<p_{c} .
$$

Then, we conclude that the collision probability in the dynamic QP approach is smaller than that in the fixed QP approach.

2) Throughput: With less collisions incurred, the dynamic QP approach leads to a higher throughput than the fixed QP approach.

Theorem 1: When the coexisting TDM and CSMA transmitters are hidden from each other, the throughput of CSMA networks under the dynamic QP approach of SHARE is higher than that under the fixed QP approach.

Proof: Recall that $1 / \lambda$ is the mean inter-arrival time between two CSMA packets. The mean service time for a CSMA packet is $1 / \lambda+l_{d}+l_{a}$.

The fixed QP approach. In this approach, the mean number of the CSMA packet arrivals during a $\mathrm{QP}$ with a length of $q f$ slots is

$$
n_{1}=\frac{q f}{\frac{1}{\lambda}+l_{d}+l_{a}} .
$$

The resulting throughput during the $i$-th $\mathrm{QP}$

$$
\phi=\frac{l_{d} n_{1}-l_{d} p_{c}}{q f}=\frac{l_{d}}{\frac{1}{\lambda}+l_{d}+l_{a}}-\frac{l_{d} p_{c}}{q f} .
$$

The dynamic QP approach. In this approach, the TDM transmitter dynamically changes the end point of the QP to reduce the probability of colliding to the last CSMA packet arrival in the QP. When the residual QP $r_{i}>l_{d}$,

- Case 1. Suppose the TDM network makes a decision of the early-termination of the QP upon an ACK detection. This early-terminated QP has a length of $\left(q_{i} f-r_{i}\right)$ slots, and the mean number of the CSMA packet arrivals during $\left(q_{i} f-r_{i}\right)$ slots is

$$
n_{2}=\frac{q_{i} f-r_{i}}{\frac{1}{\lambda}+l_{d}+l_{a}}
$$

The resulting throughput in this case

$$
\phi_{1}^{\prime}=\frac{l_{d} n_{2}-l_{d} p_{1}}{q_{i} f-r_{i}}=\frac{l_{d}}{\frac{1}{\lambda}+l_{d}+l_{a}} .
$$

We have $\phi_{1}^{\prime}>\phi$.

- Case 2. Suppose the TDM network does not make a decision of the early-termination of the QP, and wait until the end of the scheduled QP. The mean number of the CSMA packet arrivals during $q_{i} f$ slots is

$$
n_{2}^{\prime}=\frac{q_{i} f}{\frac{1}{\lambda}+l_{d}+l_{a}} .
$$

According to Lemma $1, p_{2}=p_{c}$. The resulting throughput in this case

$$
\phi_{2}^{\prime}=\frac{l_{d} n_{2}^{\prime}-l_{d} p_{2}}{q_{i} f}=\frac{l_{d}}{\frac{1}{\lambda}+l_{d}+l_{a}}-\frac{l_{d} p_{c}}{q_{i} f} .
$$

Since $q_{i} \geq q$, we have $\phi_{2}^{\prime} \geq \phi$.

The throughput under the dynamic QP approach,

$$
\phi^{\prime}=\mathbb{P}\{\text { Case } 1\} \cdot \phi_{1}^{\prime}+\mathbb{P}\{\text { Case } 2\} \cdot \phi_{2}^{\prime}>\phi .
$$

This clearly indicates that the throughput under the dynamic QP approach is higher than that under the fixed QP approach.

3) Weighted fairness: Next, we use the access time ratio (ATR) to show SHARE's property regarding the weightedfairness.

Theorem 2: Given the pre-determined values of $d$ and $q$ of the TDM super frame, the channel sharing process between TDM and CSMA MAC networks under SHARE is weightedfair.

Proof: In the $i$-th super frame, suppose the TDM MAC network makes a decision to increase the size of its $i$-th QP by $k_{i}$ frames when $\mathcal{R}_{i} \geq f$ (according the weighted-fairness maintenance algorithm (Algorithm 2)). Thus, the $i$-th QP has a length of $q_{i}=q+k_{i}$.

According the collision avoidance algorithm (Algorithm 1), the TDM MAC network is allowed to make another decision to terminate the $i$-th QP earlier than the original end, thereby leading to $e_{i}<q_{i}$. The value of $r_{i}$ quantifies the number of slots that are lost in the $i$-th QP due to this decision. Since this decision is made only when $p<\tau$. According to equation (2), we have

$$
1-e^{-\lambda\left(r_{i}-l_{d}\right)}<\tau \text {. }
$$

That is,

$$
r_{i}<\frac{\log \left(\frac{1}{1-\tau}\right)}{\lambda}+l_{d}
$$

Since $k_{i}=\frac{\mathcal{R}_{i}-\left(\mathcal{R}_{i} \bmod f\right)}{f}$ (as given in equation (3)), then

$$
\mathcal{R}_{i}-k_{i} f<f .
$$

On basis of equations (4) and (5), we have

$$
\mathcal{R}_{i+1}=\mathcal{R}_{i}+r_{i}-k_{i} f<f+\frac{\log \left(\frac{1}{1-\tau}\right)}{\lambda}+l_{d} .
$$

At the end of the $i$-th QP (i.e., the beginning of the $(i+1)$-th super frame), the TDM MAC network is able to figure out that there are a number of $\mathcal{R}_{i+1}$ slots that need to be compensated in the upcoming $\mathrm{QP}$ of the $(i+1)$-th super frame.

The ATR of the TDM MAC network, $\alpha$, is equivalent to the ratio between the length of time used for data frames and the elapsed time in all previous super frames (i.e., a total number of $i$ super frames), given by

$$
\alpha=\frac{i f d}{i f(d+q)-\mathcal{R}_{i+1}} .
$$

Using the inequality relationship of (6) in (7)

$$
\frac{i f d}{i f(d+q)}<\alpha<\frac{i f d}{i f(d+q)-f-\frac{\log \left(\frac{1}{1-\tau}\right)}{\lambda}-l_{d}} .
$$

Consequently, we have

$$
\lim _{i \rightarrow \infty} \alpha=\frac{d}{d+q} .
$$

Under SHARE, the ATR for the TDM MAC network in the long-term approximates $\frac{d}{d+q}$, which implies the weightedfairness. 


\section{Discussion}

Scalability. The quiet period (QP) mechanism is already defined in standards for TDM based networks, like 802.16 and 802.22. SHARE employs an algorithm for calculating the appropriate length of a QP, and the advanced frame technique does not affect the original scheduled data frames. Meanwhile, SHARE does not require other network configurations to be changed. Thus, the proposed protocol has little impact on the scalability of existing TDM-based network systems.

Energy consumption. In the proposed protocol, the emission of beacon and detection of ACK packets at devices in 802.22 networks will lead to increased energy consumption. However, 802.22 devices (e.g., the 802.22 base stations, and 802.22 consumer customer-premises equipment (CPE)) are not mobile devices, and are connected to wired power supplies. Given the 802.22 devices' independency of battery, the energy consumption is out of scope of this paper.

Time synchronization. It is necessary to enforce tight synchronization between base station and devices in TDM networks. There are two types of synchronization techniques: (1) Intra-cell synchronization between BS and device: the device captures the downlink control message broadcasted by the BS, obtains the super-frame and frame numbers of BS, and calculates the gap between the timers of BS and device. Synchronization can be established when the device adjusts its timer to remove the gap [15]; (2) Inter-cell synchronization between two 802.22 BSs: all base stations use a common clock derived from a global navigational systems such as GPS to synchronize their frames. Every BS derives its local clock based on the common clock. Two BSs can synchronize with each other by exchanging coexistence beacon packets (CBP) that contain the local time of each BS.

\section{Vi. Performance Evaluation}

In this section, we compare SHARE with three coexistence schemes: the centralized spectrum allocation scheme, the carrier-sensing (CS) based spectrum access scheme, and the fixed QP (FQP) mechanism. In the centralized spectrum allocation scheme, we assume a centralized controller to serve as the coordinator of coexisting TDM and CSMA networks for avoiding collisions among them. Note that the centralized scheme serves as the benchmark that represents the best performance that a coexistence scheme can achieve. In the CS based scheme, we assume that every device (including TDM device) employs a listen-before-talk protocol and only emits signals when the channel is clear. Table II presents a brief summary of the comparison results of four coexistence schemes. Next, we compare these coexistence schemes using simulation results.

\section{A. Simulation Setup}

In the simulations, we consider a heterogeneous coexistence scenario between TDM networks (e.g., 802.22 networks) and CSMA networks (e.g., 802.11 networks). In each TDM network, there is one BS and multiple TVWS devices, and the BSs of TDM networks are synchronized by a trusted third party-the 802.19.1 system. Each CSMA network is
TABLE II

The comparison of four coexistence schemes, where $p_{c}$ (previously defined as the collision probability) is the probability that the CSMA packet arrival occurs before TDM transmissions finish; $\frac{s}{f}$ is the fraction of time a device spends on spectrum sensing in the CS-based spectrum access scheme.

\begin{tabular}{|c||c|c|c|}
\hline & $\begin{array}{c}\text { Normalized } \\
\text { capacity }\end{array}$ & $\begin{array}{c}\text { Collision } \\
\text { prob. } p_{c}\end{array}$ & $\begin{array}{c}\text { Weighted } \\
\text { fairness }\end{array}$ \\
\hline \hline Centralized & $\sim 1$ & Near zero & Yes \\
\hline Carrier-sensing & $\left(1-\frac{s}{f}\right)\left(1-p_{c}\right)$ & Low & No \\
\hline FQP & $\frac{d}{d+q}\left(1-p_{c}\right)$ & High & No \\
\hline SHARE & $\frac{d}{d+q}\left(1-p_{c}\right)$ & Low & Yes \\
\hline
\end{tabular}

placed at a location such that the AP is hidden from the BS. All coexisting networks are able to identify available TVWS channels by making queries to the TVWS database or leveraging the spectrum sensing techniques.

A synchronized super frame structure is simulated for all coexisting TDM networks to share the same channel. In each data frame, we require the TDM receiver to adopts the beacon transmission mechanism to prevent the collisions to the TDM packet reception when necessary. In each quiet period, the TDM transmitters suspend their traffic and observe the possible CSMA transmission over the channel to carry out the dynamic QP mechanism. The decision-making threshold $\tau=0.5$, which implies that we require, in the residual $\mathrm{QP}$, the probability of the next complete CSMA packet transmission to be greater than the probability of an incomplete transmission. The length of QP is counted by the number of frames, and each frame contains ten time slots. The CSMA packet duration consists of multiple time slots. The CSMA packet arrival rate quantifies the probability that the CSMA packet arrives in a time slot. In the CS-based spectrum access scheme, $\frac{s}{f}=\frac{1}{5}$, which is the fraction of time a device spends on spectrum sensing in every time slot. The simulation parameter values were chosen to be consistent with those used by the 802.22 working group [16], [17].

\section{B. Mitigation of the First Type of Collisions}

We first simulate a TDM-based 802.22 network co-located with a CSMA-based WLAN sharing the same TVWS channel, where the first type of collision may occur (i.e., the scenario shown in Figure 1 when the hidden CSMA transmitter's packets collide with the packet reception at the TDM receiver). The 802.22 Working Group has suggested that a variation of the Hata model is the most appropriate propagation model for studying 802.22 networks [16]. The Hata model [25] represents the urban area propagation loss as a standard formula and supplies correction equations to model suburban and open rural areas. In our simulations, we use a variation of the Hata model for open rural areas. Suppose the distance between the TDM transmitter and receiver (with the modulation method of QPSK) is $10 \mathrm{Km}$, and the CSMA transmitter (e.g., a highpower outdoor AP) is $\delta \mathrm{Km}$ away from the TDM receiver.

SHARE enables beaconing to reduce the first type of collisions. We set the duration of beaconing $u$ as half of a time slot, which implies a 50\% performance loss as the cost for enabling the beacon transmission mechanism. By varying the value of $\delta$, we show the normalized value of channel capacity 
for the TDM network in Figure 8. When the interfering AP is close to the TDM receiver, the packet error rate is high and thus the beacon transmission is invoked to maintain a minimum value of $50 \%$ of the original channel capacity. When the distance between them increases, the interference from the hidden AP to the TDM network significantly decreases. As a result, the normalized channel capacity could be higher than $50 \%$ since the TDM network enters the non-beaconing mode. In the contrast, the FQP method does not have such a feature of beaconing, and thus the normalized channel capacity is near zero when the interferer AP is close to the TDM receiver.

The centralized scheme can always benefit from the fine coordination of the centralized control to avoid collisions among networks, and we can see the channel capacity is close to one in Figure 8. Without such a centralized controller, the CS scheme incurs a significant amount of control overhead on sensing the channel-i.e., $\frac{s}{f}$ is the fraction of time spent by a device for carrier sensing, which upper-bounds the channel capacity.

When the distance between coexisting networks increases, the interference between them decreases. In Figure 8, SHARE (or even the fixed QP approach) is able to achieve a channel capacity close to one, while the CS-based scheme cannot benefit from the decreased interference due to its control overhead spent on sensing.

\section{Mitigation of the Second Type of Collisions}

Next, we evaluate the performance of the dynamic QP mechanism in terms of the decrease of the second type of collisions.

1) Collision Ratio: We call a super frame as a collided super frame, if collisions to the CSMA packet reception are caused by the hidden TDM transmitters at the end of the QP of the super frame. We define the collision ratio as the ratio between the number of collided super frames and the number of all simulated super frames, i.e., the proportion of collided super frames.

In this set of simulations, we control the values of three parameters: the basic QP length $q$, the CSMA packet duration $l_{d}$, and the CSMA packet arrival rate $\lambda$. We fixed the values of two parameters, and vary the value of the third one. Since we only focus on the collisions caused by the hidden terminals at the end of each QP, the increase of the basic QP length only causes a negligible change in the collision ratio under all of the coexistence schemes, as shown in Figure 9. Moreover, the FQP approach leads to the highest collision ratio, while the others can effectively mitigate the collision problem with a small collision ratio that is invulnerable to the change of $\mathrm{QP}$ length.

With an increased CSMA packet duration $l_{d}$ or an increased the packet arrival rate $\lambda$, the probability that a CSMA transmission is unfinished at the end of the fixed QP increases as well. Under the fixed QP approach, the TDM transmitter will be more likely to cause collisions to the ongoing CSMA transmission. In SHARE, the TDM transmitter is free to terminate the QP dynamically based on its observation, which could effectively mitigate this type of collisions. Hence, both
Figures 10 and 11 shows an increased collision ratio for the FQP approach and the CS-based scheme, while only a nearlyinvariant collision ratio is measured for SHARE.

2) Normalized Throughput in the Quiet Period: In a quiet period based approach, the packet collision due to the hidden terminals only happens at the end of a QP.

We define the normalized throughput in the $Q P$ as the ratio between the number of time slots for transmitting CSMA packets without collisions during a QP and the total number of time slots in the QP. In this set of simulations, we observe the normalized throughput under various combinations of parameter values. In general, Figures 12, 13, and 14 show that the performance of SHARE is better than that of the FQP scheme in all cases.

As shown in Figure 12, the gap between SHARE and FQP in terms of the normalized throughput narrows as the length of the QP increases. In contrary, the throughput gap between SHARE and FQP becomes wider when we increase the CSMA packet duration $l_{d}$, as shown in Figure 13. The reason has been explained above (for Figure 10): with an increased CSMA packet duration, the collision probability increases in every QP, which decreases the normalized throughput. Another main contributing factor to the increase of throughput is the CSMA packet arrival rate, as we can observe from results in Figure 14.

The purpose of this set of simulations is to investigate how much is the performance gain that can be obtained from the proposed protocol. We evaluated the protocol by varying three factors, including the length of the quiet period, the CSMA packet duration, and CSMA packet arrival rate.

- In Figure 12, the performance gain is approximately $20 \%$ when the length of the quiet period is as small as the half of a frame length;

- As Figure 13 shows, the longer the CSMA packet is, the more performance gain can be obtained (approximately $40 \%$ when the CSMA packet length is 5 slots).

- In Figure 14, the performance gain is insignificant (approximately 5-10\%) in all cases with different packet arrival rates.

Thus, the proposed protocol has a significant performance gain when a CMSA network that has a long packet length coexists with a TDM network that has a small QP length. Meanwhile, it has no significant performance gain given various packet arrival rates.

3) Weighted-fairness: In this simulation, we vary the basic QP length, and in each simulation run, we set $l_{d}=2$ and $\lambda=0.5$. We measure the access time ratio for the TDM network under two approaches: SHARE's dynamic QP approach without the weighted-fairness maintenance (WM) mechanism, and SHARE's dynamic QP approach with the WM mechanism. The group of white bars in Figure 15 represents the pre-determined values of the TDM networks' access time ratio. The group of light-grey bars clearly show that SHARE with WM allocates spectrum in a weighted-fair manner, and it has an advantage of guaranteeing the access time ratio close to the pre-determined value. 


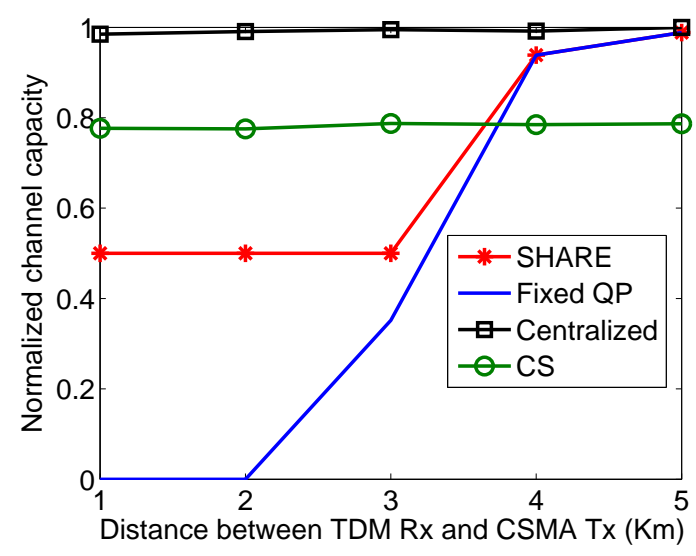

Fig. 8. Normalized channel capacity, where $\frac{s}{f}=\frac{1}{5}$.

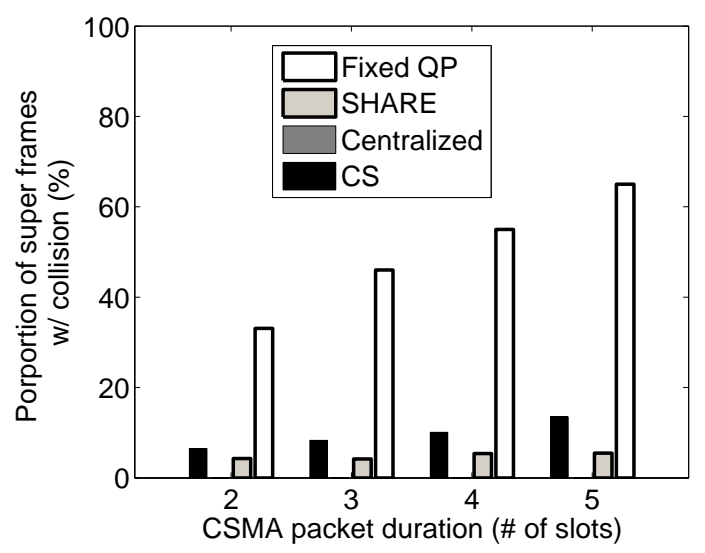

Fig. 10. Collision ratio when varying the CSMA packet duration, $q=1$ and $\lambda=0.5$.

\section{RELATED WORK}

In this section, we give a brief overview on the existing coexistence schemes.

The homogeneous coexistence problem between coexisting 802.22 networks is also known as self-coexistence problem. Since the inter-BS communication is feasible in IEEE 802.22, the coordinated coexistence scheme is a preferred option. The homogeneous coexistence problem can be addressed via a game theoretic approach [4], [19], [26], or a channel contention procedure [10]. Note that the communication between homogeneous networks does not rely on a third party, while the coordination between heterogeneous networks does require a third party (e.g., the 802.19.1 system) as a mediator.

Autonomous coexistence schemes also work for addressing the homogeneous coexistence problem of uncoordinated $\mathrm{WiFi}$ deployments and femto cell deployments. Without the inter-AP coordination method, channel hopping sequences are designed to avoid the interference based on the WLAN channel observation [20]. In OFDMA femto cells, the spectrum can be allocated to the macro BS and the femto BS in a distributed mannerthe hash function is used to reduce collisions when the same OFDMA block is allocated to multiple users [30]. CTRL is

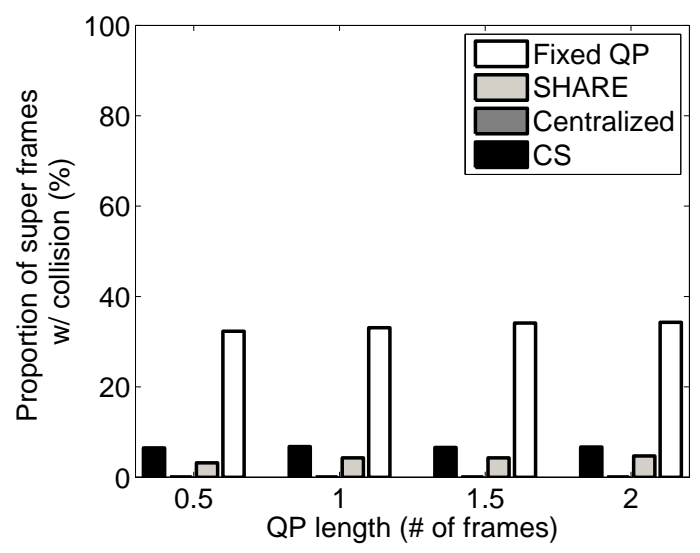

Fig. 9. Collision ratio (proportion of collided super frames) under the hidden terminal situation, when varying the basic QP length, $l_{d}=2$ and $\lambda=0.5$.

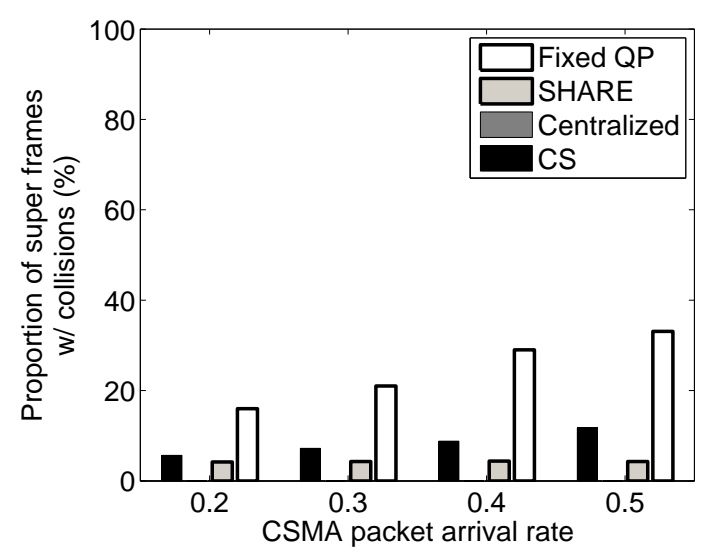

Fig. 11. Collision ratio when varying the CSMA packet arrival rate, $q=1$ and $l_{d}=2$.

a self-organizing femtocell management architecture, which controls the femto cell's transmission power for protection of macrocell communications from interference, and the efficient coordination of the resource usage among femtocells [34].

To address the problem of coexistence among wireless networks in TVWS, a standard-independent framework is proposed to enable exchange of information relevant for coexistence, which includes two types of approaches: centralized and distributed [32]. The introduction of a multi-radio clusterhead equipment (CHE) as a physical entity simplifies the implementation of this framework. In the centralized approach, coexisting networks refer to databases for coexistence-related information. The distributed approach leverages the multiradio feature of CHE to distribute beacons and coordinate with neighboring non-compliant networks over a common broadcast channel.

\section{CONCLUSIONS}

In this paper, we investigate the hidden terminal problem in the context of heterogeneous coexistence, and present an autonomous coexistence protocol, called SHARE, that mitigates this problem between TDM-based networks and CSMAbased networks. SHARE enables the channel sharing between 


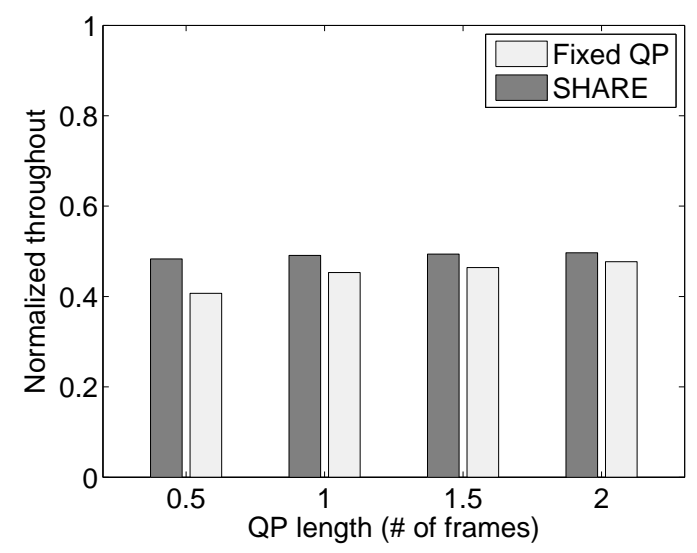

Fig. 12. Normalized throughput under the hidden terminal situation, when varying the basic QP length, $l_{d}=2$ and $\lambda=0.5$.

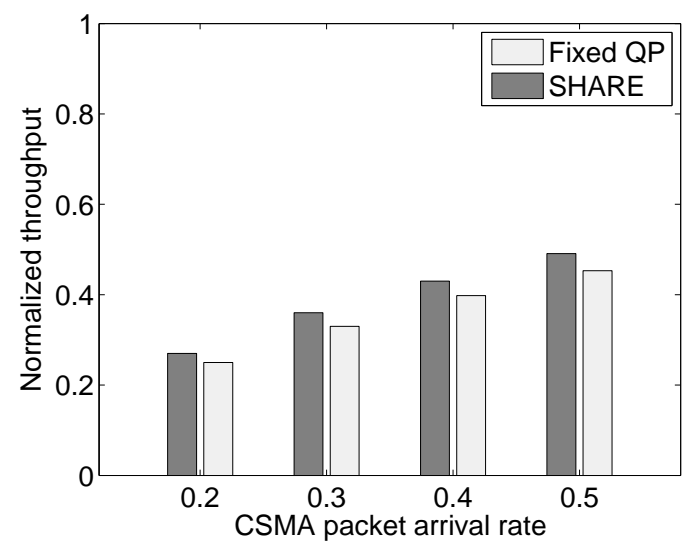

Fig. 14. Normalized throughput when varying the CSMA packet arrival rate, $q=1$ and $l_{d}=2$.

heterogeneous networks by employing two mechanisms. The beacon transmission mechanism allows the TDM receivers to prevent the hidden CSMA transmitters from interfering with TDM data receptions. To avoid interfering to CSMA network's data receptions, the TDM network adopts a dynamic quiet period mechanism that consists of two algorithms: (1) a collision avoidance algorithm for the TDM network to dynamically determine the end point of the quiet period so as to reduce the collision probability that are introduced by the hidden terminals; and (2) a weighted fairness maintenance algorithm for adjusting the length of future quiet periods to fairly divide the channel access time among the coexisting networks. SHARE is independent of the coordination between heterogeneous networks, and it requires no change to the CSMA networks. Compared with the fixed quiet period approach, SHARE guarantees a reduced collision probability, a better throughout for CSMA networks, and the longer-term weighted fairness.

\section{REFERENCES}

[1] P. Bahl, R. Chandra, T. Moscibroda, R. Murty, and M. Welsh. White Space Networking with Wi-Fi like Connectivity. Proc. of Sigcomm, pp. 27-38, August 2009.

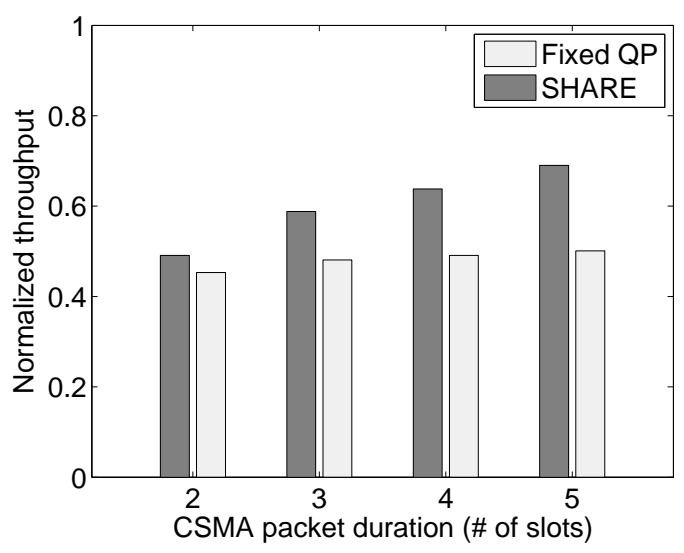

Fig. 13. Normalized throughput when varying the CSMA packet duration, $q=1$ and $\lambda=0.5$.

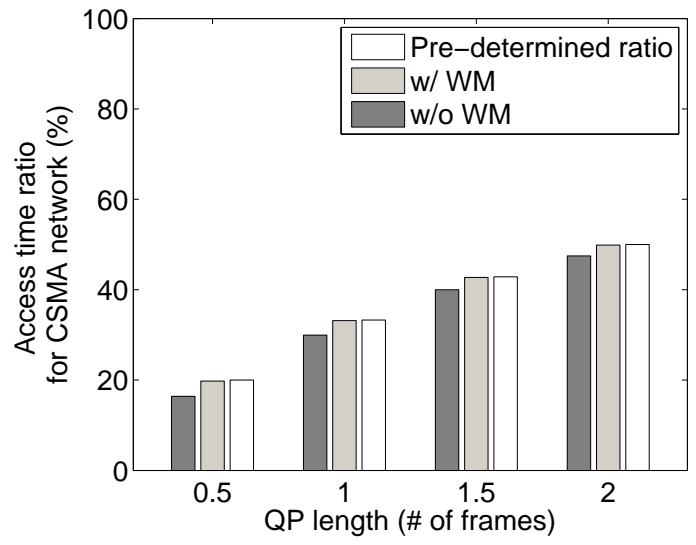

Fig. 15. The access time ratio for the TDM network.

[2] T. Baykas, M. Cummings, H. Kang, M. Kasslin, J. Kwak, Richard Paine, A. Reznik, R. Saeed, and S. J. Shellhammer. Developing a Standard for TV White Space Coexistence: Technical Challenges and Solution Approaches. IEEE Wireless Communications, 19(1):10-22, Feb. 2012.

[3] L. Berlemann, C. Hoymann, G. Hiertz, and B. Walke. Unlicensed Operation of IEEE 802.16: Coexistence with 802.11(A) in Shared Frequency Bands. Proc. of PIMRC, pp. 1-5, September 2006.

[4] S. Brahma and M. Chatterjee. Mitigating Self-Interference Among IEEE 802.22 Networks: A Game Theoretic Perspective. Proc. of IEEE Globecom, pp. 1-6, November 2009.

[5] F. Daneshgaran, M. Laddomada, F. Mesiti, and M. Mondin. On the Linear Behaviour of the Throughput of IEEE 802.11 DCF in NonSaturated Conditions. IEEE Communications Letters, 11(11):856-858, November 2007.

[6] Ecma International. ECMA-392: MAC and PHY for Operation in TV White Space. 1st Edition, December 2009.

[7] X. Fu, W. Ma, and Q. Zhang. The IEEE 802.16 and 802.11a Coexistence in the License-Exempt Band. Proc. of IEEE WCNC, pp. 1942-1947, October 2007.

[8] B. Gao, J. Park, Y. Yang, and S. Roy. A Taxonomy of Coexistence Mechanisms for Heterogeneous Cognitive Radio Networks Operating in TV White Spaces. IEEE Wireless Communications 19(4): 41-48, August 2012.

[9] C. Ghosh, S. Roy, and Cavalcanti. Coexistence Challenges for Heterogeneous Cognitive Wireless Networks in TV White Spaces. IEEE Wireless Communications 18(4): 22-31, August 2011.

[10] D. Grandblaise and W. Hu. Inter Base Stations Adaptive On Demand Channel Contention for IEEE 802.22 WRAN Self Coexistence IEEE docs: IEEE 802.22-07/0024r0, January. 2007.

[11] J. Huang, G. Xing, G. Zhou, and R. Zhou. Beyond Co-existence: 
Exploiting WiFi White Space for ZigBee Performance Assurance. Proc. of IEEE ICNP, pp. 305-314, October 2010.

[12] X. Jing, and D. Raychaudhuri. Spectrum co-existence of IEEE $802.11 \mathrm{~b}$ and 802.16a networks using the CSCC etiquette protocol. Proc. of IEEE Dyspan, pp. 243-250 , November 2005.

[13] IEEE 802.19 Task Group 1. Wireless Coexistence in the TV White Space. http://www.ieee802.org/19/pub/TG1.html.

[14] IEEE 802.19 Task Group 1. Coexistence in the TV White Space. IEEE doc.: 802.19-10/009r6.

[15] IEEE 802.22 Working Group. IEEE Standard for Wireless Regional Area Networks Part 22: Cognitive Wireless RAN Medium Access Control (MAC) and Physical Layer (PHY) Specifications: Policies and Procedures for Operation in the TV Bands. IEEE Standard 802.22, July 2011.

[16] IEEE 802.22 Working Group. Reviews of Channel Model. IEEE docs: 22-05-0070-00-0000, August 2005.

[17] IEEE 802.22 Working Group. ETRI FT Philips Samsung Proposal. IEEE docs: 22-06-0005-01-0000, Jan. 2006.

[18] P. Karn. MACA-A New Channel Access Method for Packet Radio. Proc. of ARRL/CRRL Amateur Radio 9th Computer Networking Conference, pp. 134-140, 1990.

[19] C. Ko and H. Wei. Game Theoretical Resource Allocation for InterBS Coexistence in IEEE 802.22. IEEE Transactions on Vehicular Technology, 59(4):1729-1744, May 2010.

[20] A. Mishra, V. Shrivastava, D. Agarwal, S. Banerjee and S. Ganguly. Distributed Channel Management in Uncoordinated Wireless Environments. Proc. of ACM MobiCom, pp. 170-181, September 2006.

[21] R. Murty, R. Chandra, T. Moscibroda, and P. Bahl. SenseLess: A Database-driven White Spaces Network. Proc. of IEEE DySpan, pp. 10-21, May 2011.

[22] P. Piggin. An Overview of $802.16 \mathrm{~h}$ 'Uncoordinated' Coexistence Approach in 3.65-3.7GHz. S80216h-06_117, November, 2006.

[23] J. Proakis and M. Salehi. Communication Systems Engineering. Prentice Hall, Second Edition, 2001

[24] B. Radunovic, R. Chandra, and D. Gunawardena. Adaptive Preambles for Coexistence. Micorsoft technical report, MSR-TR-2011-15, January 2011.

[25] T. S. Rappaport. Wireless Communications: Principles and Practice. Upper Saddle River, NJ: Prentice Hall, 2001.

[26] S. Sengupta, R. Chandramouli, S. Brahma, and M. Chatterjee. A Game Theoretic Framework for Distributed Self-Coexistence among IEEE 802.22 Networks. Proc. of IEEE Globecom, November 2008.

[27] S. J. Shellhammer. Estimation of Packet Error Rate Caused by Interference using Analytic Techniques-A Coexistence Assurance Methodology. IEEE docs: P802.19-05/0028r1, September 2005.

[28] M. M. Siddique, B.-L. Wenning, and M. Muehleisen. Spectrum Sharing between IEEE 802.16 and IEEE 802.11 based Wireless Networks. IEEE WoWMoM, pp. 1-6, June 2010

[29] J. So. and N. H. Vaidya. Multi-channel MAC for Ad Hoc Networks: Handling Multi-channel Hidden Terminal using A Single Transceiver. Proc. of ACM MobiHoc, pp. 222-233, May 2004.

[30] K. Sundaresan and S. Rangarajan. Efficient Resource Management in OFDMA Femto Cells. Proc. of ACM MobiHoc, pp. 33-42, May 2009.

[31] N. Thomas, M. Willis, and K. Craig. Analysis of Co-existence between IEEE 802.11 and IEEE 802.16 Systems. IEEE SECON, pp. 615-620, 2006.

[32] G.P. Villardi, Y.D. Alemseged, C. Sun; C.-S. Sum; T. Nguyen; T. Baykas, and H. Harada. Enabling Coexistence of Multiple Cognitive Networks in TV White Space. IEEE Wireless Communications, 18(4):32-40, August 2011.

[33] Wireless LAN Medium Access Control (MAC) and Physical Layer (PHY) Specifications. IEEE standard. 802.11, 2007.

[34] J. Yun and K. Shin. CTRL: A Self-organizing Femtocell Management Architecture for Co-channel Deployment. Proc. of ACM MobiCom, pp. 61-72, September, 2010.

[35] X. Zhang and K. G. Shin. Enabling Coexistence of Heterogeneous Wireless Systems: Case for ZigBee and WiFi. Proc. of ACM MobiHoc, May 2011.

[36] R. Zhou, Y. Xiong, G. Xing, L. Sun, and J. Ma. ZiFi: Wireless LAN Discovery via ZigBee Interference Signatures. Proc. of ACM Mobicom, pp. 49-60, September 2010.

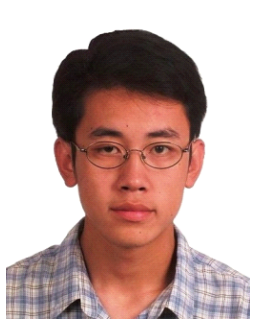

Kaigui Bian received his Ph.D. degree in computer engineering from Virginia Tech, Blacksburg, USA in 2011. He is currently an assistant professor in the Institute of Network Computing and Information Systems, School of EECS at Peking University. His research interests include mobile computing, cognitive radio networks, network security and privacy.

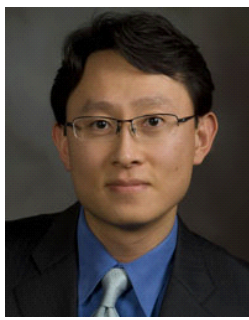

Jung-Min "Jerry" Park received a Ph.D. degree in electrical and computer engineering from Purdue University in 2003 . He is currently an associate professor in the Department of Electrical and Computer Engineering at Virginia Tech, and the site director of a National Science Foundation (NSF) IndustryUniversity Cooperative Research Center (I-UCRC) called Broadband Wireless Access \& Applications Center (BWAC). As the site director of BWAC at Virginia Tech, Park is leading several sponsored research projects on wireless networks and network security. He is widely recognized for his pioneering work on enforcement and security problems in cognitive radio networks. His research interests include cognitive radio networks, spectrum sharing technologies, network security and privacy, and applied cryptography. Current or recent research sponsors include the NSF, National Institutes of Health (NIH), Defense Advanced Research Projects Agency (DARPA), Office of Naval Research (ONR), SANS (SysAdmin, Audit, Network Security) Institute, Motorola Solutions, Samsung Electronics, and SCA Techniques. More details on his research interests can be found at http://www.arias.ece.vt.edu. He is a recipient of a 2008 NSF Faculty Early Career Development (CAREER) Award, a 2008 Hoeber Excellence in Research Award, and a 1998 AT\&T Leadership Award. He is a senior member of the IEEE and the ACM, and a member of the Korean-American Scientists and Engineers Association (KSEA).

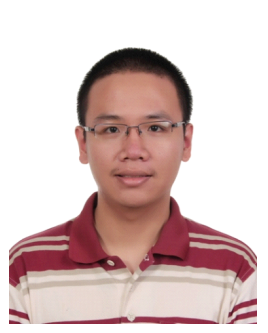

Lin Chen is currently an undergraduate student in the school of EECS at Peking University. His research interests focus on wireless networks and network theory.

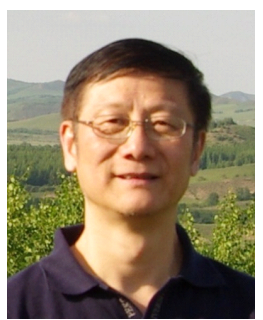

Xiaoming $\mathbf{L i}$ received his $\mathrm{Ph} . \mathrm{D}$. in computer science from Stevens Institute of Technology (USA) in 1986 and now is a Professor at Peking University. His research interests include Web search and mining, online social network analysis. He is an editor of Concurrency and Computation, and an editor of Networking Science. 